\title{
Modeling nonthermal emission from stellar bow shocks (Research Note)
}

\author{
V. Pereira ${ }^{1}$, J. López-Santiago ${ }^{1}$, M. Miceli ${ }^{2,3}$, R. $^{\text {Bonito }}{ }^{2,3}$, and E. de Castro ${ }^{1}$ \\ 1 Dpto. de Astrofísica y CC de la Atmósfera, Universidad Complutense de Madrid, 28040 Madrid, Spain \\ e-mail: vpereirablanco@ucm.es \\ 2 INAF-Osservatorio Astronomico di Palermo, Piazza del Parlamento 1, 90134 Palermo, Italy \\ ${ }^{3}$ Dipartimento di Fisica e Chimica, Università di Palermo, Piazza del Parlamento, 90134 Palermo, Italy
}

Received 16 December 2015 / Accepted 16 February 2016

\begin{abstract}
Context. Runaway O- and early B-type stars passing through the interstellar medium at supersonic velocities and characterized by strong stellar winds may produce bow shocks that can serve as particle acceleration sites. Previous theoretical models predict the production of high-energy photons by nonthermal radiative processes, but their efficiency is still debated.

Aims. We aim to test and explain the possibility of emission from the bow shocks formed by runaway stars traveling through the interstellar medium by using previous theoretical models.

Methods. We applied our model to AE Aurigae, the first reported star with an X-ray detected bow shock, to BD+43 3654, in which the observations failed in detecting high-energy emission, and to the transition phase of a supergiant star in the late stages of its life. Results. From our analysis, we confirm that the X-ray emission from the bow shock produced by AE Aurigae can be explained by inverse Compton processes involving the infrared photons of the heated dust. We also predict low high-energy flux emission from the bow shock produced by $\mathrm{BD}+433654$, and the possibility of high-energy emission from the bow shock formed by a supergiant star during the transition phase from blue to red supergiant.

Conclusions. Bow shocks formed by different types of runaway stars are revealed as a new possible source of high-energy photons in our neighborhood.
\end{abstract}

Key words. acceleration of particles - radiation mechanisms: non-thermal - shock waves - X-rays: ISM

\section{Introduction}

Runaway stars are stars that exceed the typical velocities of the stars in the galaxy and can reach more than $200 \mathrm{~km} \mathrm{~s}^{-1}$ (e.g., Blaauw 1961; Gies \& Bolton 1986). To distinguish them from the stars with typical velocities and dispersions of about $10 \mathrm{~km} \mathrm{~s}^{-1}$, runaway stars are considered to be those with $V_{*}>$ $30 \mathrm{~km} \mathrm{~s}^{-1}$. When these stars move supersonically through the interstellar medium (ISM) and the medium density is high enough, the stellar wind sweeps the circumstellar dust together and piles it up, leading to the formation of a bow shock in the direction of their movement.

Relativistic particles can be accelerated by strong shocks, and the interaction of these particles with the matter, the radiation, and the magnetic field of the bow shock region can produce nonthermal emission. Benaglia et al. (2010) detected radio emission from the bow shock produced by the runaway star BD+43 3654, consistent with synchrotron radiation produced by the interaction of the relativistic electrons with the magnetic field of the acceleration region. Later, del Valle \& Romero (2012) presented a nonthermal emission model that predicted the production of high-energy photons in these acceleration regions in sufficiently large numbers to be detected in X-rays. López-Santiago et al. (2012) published the first detection of X-ray emission from a bow shock produced by a runaway star (AE Aurigae), while Terada et al. (2012) reported the non-detection of X-ray emission in the bow shock region formed by BD+433654 from a long-duration X-ray observation with Suzaku.
As a result of their strong winds, $\mathrm{O}$ and early-B runaway main-sequence-type stars have been traditionally considered to be the best candidates to form strong bow shocks. With masses higher than $10 M_{\odot}$ and temperatures between 10 and $50 \mathrm{kK}$, these stars lose mass at a rate of $\dot{M} \sim 10^{-7}-10^{-5} M_{\odot} \mathrm{yr}^{-1}$ in their short life $(\leq 20 \mathrm{Myr})$, and their stellar winds, with velocities ranging from 1000 to $3000 \mathrm{~km} \mathrm{~s}^{-1}$, transfer great amounts of mechanical energy to the circumstellar medium. Searches of runaway O and early-B have been performed by Gies \& Bolton (1986), Gies (1987), Moffat et al. (1998, 1999) and Maíz-Apellániz et al. (2004). More recently, an extensive kinematic and probabilistic study has led to the identification of 2500 candidate runaway stars from the data of the HIPPARCOS catalog (Tetzlaff et al. 2010).

In this work we aim to explain the X-ray emission from the bow shock formed by AE Aurigae and the non X-ray detection from the bow shock of BD+43 3654, and also to extend the study to other runaway stars interacting with the ISM. For this purpose, we applied the model derived by del Valle \& Romero (2012), where different nonthermal processes are proposed to explain the origin of the emission in these shocks. Further details on the nonthermal processes are also found in Adams (1980), Kelner et al. (2006), Longair (2011) or Rybicki \& Lightman (1979). Other contributions to explain the high-energy emission from the bow shocks produced by runaway stars are those of Benaglia et al. (2010), Terada et al. (2012), and del Valle \& Romero (2014). 
Table 1. Model parameters.

\begin{tabular}{|c|c|c|c|c|}
\hline \multicolumn{5}{|c|}{ Stellar parameters } \\
\hline & Parameters & AE Aurigae & $\mathrm{BD}+433654$ & Betelgeuse \\
\hline$\dot{M}$ & Wind mass-loss rate $\left[M_{\odot} \mathrm{yr}^{-1}\right]$ & $10^{-7}$ & $10^{-5}$ & $3.2 \times 10^{-7}$ \\
\hline$V_{\mathrm{w}}$ & Wind velocity $\left[\mathrm{km} \mathrm{s}^{-1}\right]$ & 1500 & 2300 & 270 \\
\hline$R_{0}$ & Standoff radius $[\mathrm{pc}]$ & 0.082 & 1.5 & 0.63 \\
\hline$L_{\star}$ & Luminosity of the star $\left[L_{\odot}\right]$ & $0.7 \times 10^{5}$ & $8 \times 10^{5}$ & $4.8 \times 10^{4}$ \\
\hline$T_{\star}^{\star}$ & Stellar temperature $[\mathrm{kK}]$ & 32 & 39 & 9 \\
\hline$R_{\star}^{\star}$ & Stellar radius $\left[R_{\odot}\right]$ & 8.9 & 19.4 & 90 \\
\hline $\begin{array}{l}n_{\star} \\
n_{\mathrm{H}}\end{array}$ & Ambient medium density $\left[\mathrm{cm}^{-3}\right]$ & 2.3 & 5 & 1.3 \\
\hline$V_{\star}$ & Stellar velocity $\left[\mathrm{km} \mathrm{s}^{-1}\right]$ & 150 & 66 & 50 \\
\hline$d{ }^{\star}$ & Distance $[\mathrm{pc}]$ & 550 & 1450 & 200 \\
\hline Age & Bow shock lifetime [Myr] & 2.5 & 1.6 & 30 \\
\hline \multicolumn{5}{|c|}{ Free parameters in the model } \\
\hline$\Delta$ & Shock width $\left[R_{0}\right]$ & 0.3 & 0.08 & 0.25 \\
\hline$\alpha$ & Electron injection index & 2 & 2.1 & 2 \\
\hline$q_{\text {rel }}$ & Fraction of relativistic energy & 0.15 & 0.1 & 0.1 \\
\hline$a$ & Electron-to-proton ratio & 1 & 1 & 1 \\
\hline
\end{tabular}

\section{Applications}

López-Santiago et al. (2012) reported the first X-ray detection of a bow shock formed by a runaway star (AE Aurigae). Terada et al. (2012) did not succeed in detecting significant diffuse X-rays from the bow shock formed by the runaway star $\mathrm{BD}+433654$. In this section, we apply our nonthermal model to clarify the origin of the X-ray emission detected from the bow shock formed by the star AE Aurigae (HIP 24575), and to try to explain the non-detection in this band of the bow shock formed by $\mathrm{BD}+433654$. We then extend our study to other stars to test wether the bow shocks they form can also be a source of highenergy photons in our galaxy. In particular, we study the bow shock formed by a supergiant star during the transition from blue to red using the star Betelgeuse as a test of our computations.

The power available in the acceleration region of a bow shock is given by the volume ratio, that is, $L=V L_{T} / V_{R_{0}}$, where $L_{T}$ is the kinetic energy released by the stellar wind per second, $V_{R_{0}}$ is the volume of a sphere of radius $R_{0}$, and $V$ is the volume of the acceleration region. $R_{0}$ is the so-called standoff radius expressed by (Wilkin 1996)

$R_{0}=\sqrt{\frac{\dot{M} V_{\mathrm{w}}}{4 \pi \rho_{\mathrm{ISM}} V_{\star}^{2}}}$.

Here, $\dot{M}$ is the mass-loss rate, $V_{\mathrm{w}}$ the wind velocity, $\rho_{\mathrm{ISM}}\left(\rho_{a}=\right.$ $n_{\mathrm{H}} \mu$, where $n_{\mathrm{H}}$ is the ambient column density in $\mathrm{cm}^{-3}$ and $\mu=$ $2.3 \times 10^{-24} \mathrm{~g}$ the mass per $\mathrm{H}$ atom) is the ambient density, and $V_{\star}$ is the stellar velocity. We assume that only a small fraction $q_{\text {rel }}$ of $L$ is turned into relativistic particles and thus the total energy of the accelerated charged particles is $L_{\mathrm{rel}}=q_{\mathrm{rel}} L$. These relativistic particles can either be electrons or protons, which means that $L_{\text {rel }}$ has both a leptonic and a hadronic component, $L_{\mathrm{rel}}=L_{\mathrm{p}}+L_{\mathrm{e}}=$ $a L_{\mathrm{e}}+L_{\mathrm{e}}$, where $a$ is the ratio of relativistic protons to electrons.

In the three simulations we assumed the medium the stars are traveling through to be homogeneous and free of clumps and that the IC seed fields are isotropic. In Table 1 we list the stellar parameters of these three stars and the free parameters we used. The electron-to-proton ratio, although a free parameter of the model, was fixed to $a=1$ (see explanation in Sect. 2.1). Both the electron injection index and the fraction of relativistic energy were fitted to the available observational data points. The IR shock widths were taken from Peri et al. (2012). However, the nonthermal emission is produced from a smaller region, concentrated in a small knot at the bow shock apex, and hence the final shock width was also obtained from fitting the observational data.

\subsection{AE Aurigae (AE Aur)}

The runaway star AE Aur was ejected from the Orion nebula cluster after an encounter between two systems about 2.5 million years ago. It is considered a runaway star for its high velocity, $v_{*} \approx 150 \mathrm{~km} \mathrm{~s}^{-1}$ (Peri et al. 2012). As a result of this interaction, $\mathrm{AE}$ Aur and $\mu \mathrm{Col}$ (both O9.5 type stars) were expelled at great velocities, while $\iota$ Ori remained forming an eccentric binary system with the two more massive stars (Hoogerwerf et al. 2000). In its path, AE Aur encountered the dense molecular cloud IC 405, with a density $n_{\mathrm{H}} \sim 3 \mathrm{~cm}^{-3}$ (see Peri et al. 2012). The interaction between the strong stellar wind and the dust resulted in the formation of a bow shock, which was first detected in the mid-IR by Van Buren \& McCray (1988) using IRAS. The terminal wind velocity of the star is $v_{\mathrm{w}} \approx 1500 \mathrm{~km} \mathrm{~s}^{-1}$ (Hubrig et al. 2011) and its mass-loss rate $\dot{M} \approx 10^{-7} M_{\odot} \mathrm{yr}^{-1}$ (Fullerton et al. 2006). The cooling time rates obtained for these parameters are represented in Fig. 1.

Figure 3 shows the synthesized luminosity spectrum of the bow shock formed by the star AE Aur. The X-ray emission from the bow shock reported by López-Santiago et al. (2012) is concentrated in a small knot northeast of the star and can be explained by the IC emission of the heated dust, with a very small contribution of the IC of the starlight. The synchrotron emission is high at radio frequencies, and the IR is dominated by the thermal emission. The thermal and the relativistic Bremsstrahlung are negligible compared to the IC of the dust and to the IC of the starlight photons, The IC of the CMB is very faint. The XMM-Newton EPIC pn data obtained by López-Santiago et al. (2012) are included, and the Fermi detection limit from Acero et al. (2015) is also shown.

The hadronic emission produced by the proton-proton inelastic collisions is negligible, revealing that the energy released during the shock interaction is too low to produce high-energy hadronic emission.

On the other hand, if we consider $a=100$, as in the cosmic rays case (Ginzburg \& Syrovatskii 1964), we cannot reproduce 
V. Pereira et al.: Modeling nonthermal emission from stellar bow shocks $(R N)$

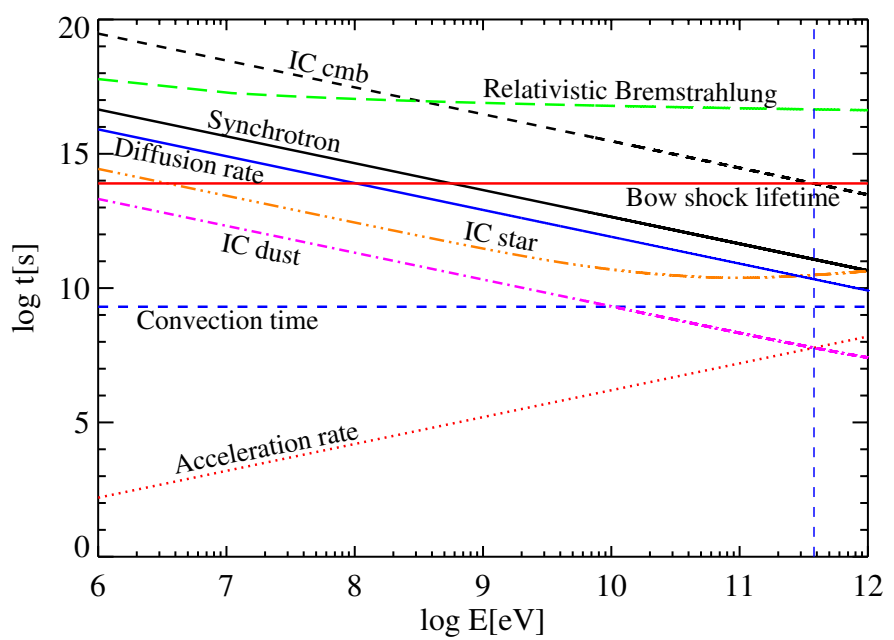

Fig. 1. Leptonic cooling time rates, acceleration rate, convection time, diffusion rate and bow shock lifetime for the parameters of the bow shock formed by AE Aurigae (see Sect. 2.1). The vertical dashed line indicates the maximum energy.

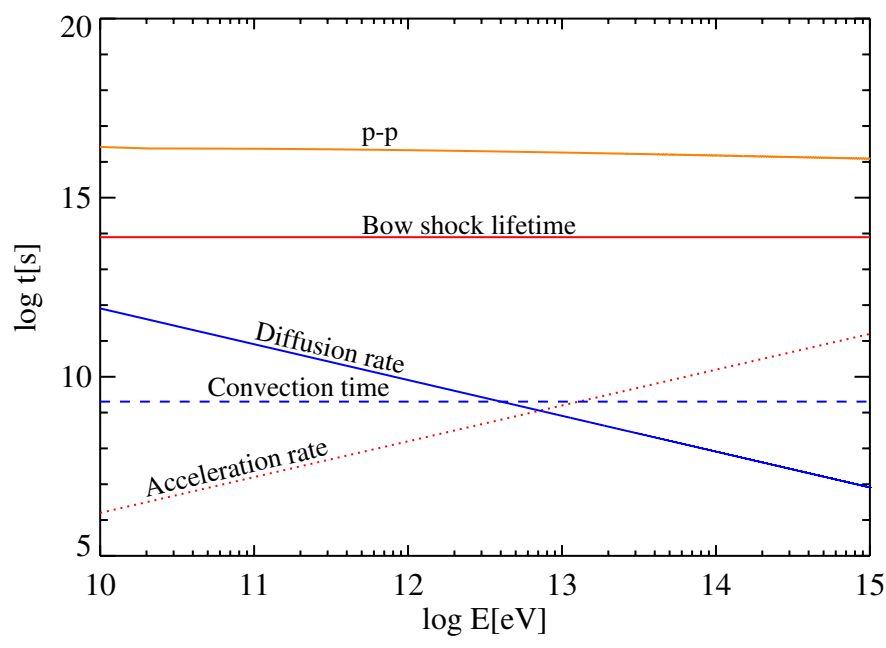

Fig. 2. Proton-proton inelastic collision time rate, convention time, diffusion rate, and bow shock lifetime for the parameters of the bow shock formed by AE Aurigae (see Sect. 2.1). The cooling time rates represent the time a particle with energy $E$ needs to cool completely by a given process.

the X-ray emission detected. In Fig. 2 we showed that there are not enough p-p collisions and hence no secondary electrons are generated that can produce the required IC flux to explain the $\mathrm{X}$-ray emission. In contrast, because for $a=100$ the energy that goes into electrons is dramatically reduced, the primary electrons are unable to reproduce the X-ray flux observed.

\section{2. $B D+433654$}

Comerón \& Pasquali (2007) concluded that the origin of the high velocity of the star BD+343654 was the dynamical interaction between two massive binaries. In contrast, Gvaramadze \& Bomans (2008) determined that this star was formed from the stellar fusion of two stars after the encounter of two binary systems, originally with $\sim 35$ and $\sim 50-60 M_{\odot}$, respectively. The high linear momentum of the star indicates that the encounter was very energetic. The most massive object is now seen as an O4If.

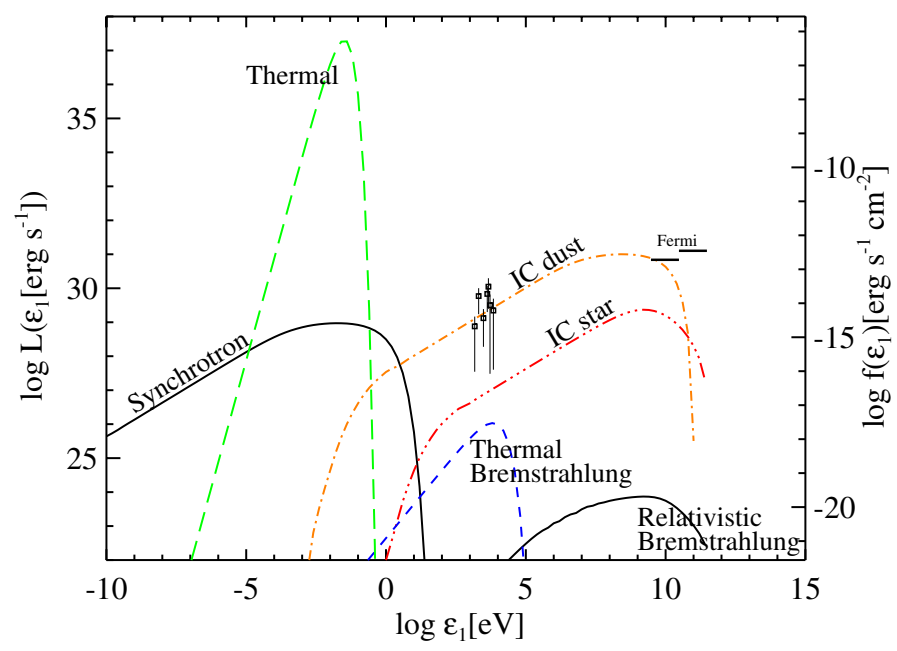

Fig. 3. Synthesized luminosity spectrum for the bow shock formed by the star AE Aur. The Fermi detection limit from Acero et al. (2015) and the XMM-Newton data points are also included.

BD+43 3654 has been reported to have formed a bow shock detected in the IR (Comerón \& Pasquali 2007) and in radio (Benaglia et al. 2010). These two observations are coincident and extensive.

Assuming the velocity of this star to be $67 \mathrm{~km} \mathrm{~s}^{-1}$ (Kobulnicky et al. 2010), the mass-loss rate $10^{-5} M_{\odot} \mathrm{yr}^{-1}$ (Markova et al. 2004; Repolust et al. 2004), the stellar wind velocity $2300 \mathrm{~km} \mathrm{~s}^{-1}$ (Howarth et al. 1997), and the standoff radius $R_{0}=1.5$ pc (Peri et al. 2012), we derive from Eq. (1) a medium density of $5 \mathrm{~cm}^{-3}$. The extensive emission in the radio and IR wavelengths are found at a distance consistent with $R_{0}$.

$\mathrm{BD}+433654$ has one of the greatest mass-loss rates among the $\mathrm{O}$ and $\mathrm{B}$ type stars (see Vink et al. 2001), and the same is true for the stellar wind (see Howarth et al. 1997). This great amount of released energy and a moderate velocity allow the bow shock to be formed far away from the star, although the energy is so high that the star could have crossed the ISM without forming a strong shock. However, the radio and IR detections discard this possibility. In this case, the high density of the interstellar region crossed by the star favors the formation of the shock. When these considerations about the stellar parameters of this star are taken into account, the values in the nominator of Eq. (1) are revealed to be among the highest of all the stars known. In contrast, the value of the velocity of this star, in the denominator, is moderate. Under these circumstances, a higher velocity would lead to a closer shock, whereas lower velocities would mean that $\mathrm{BD}+433654$ is not a runaway star. With such parameters, the bow shock formed by this star possibly represents an upper limit for the standoff radius of a bow shock formed by a runaway star. The radio and IR points allowed us to fit the spectrum and the values of the free parameters.

The spectrum of the bow shock derived from our model is shown is Fig. 4. Benaglia et al. (2010) suggested that the detection of VLA radio emission is associated with the cooling of electrons by synchrotron emission. However, from our computations the radio points can be explained by the sum of the nonthermal synchrotron emission and the thermal emission of the shocked dust, also consistent with the MSX data at $0.1 \mathrm{eV}$. Here the IC of the dust photons, plus the thermal Bremsstrahlung, hardly reach the XMM-Newton detection limit proposed by Hasinger et al. (2001) for $100 \mathrm{ks}$. This suggests that the bow shock of BD+43 3654 could be detectable through 


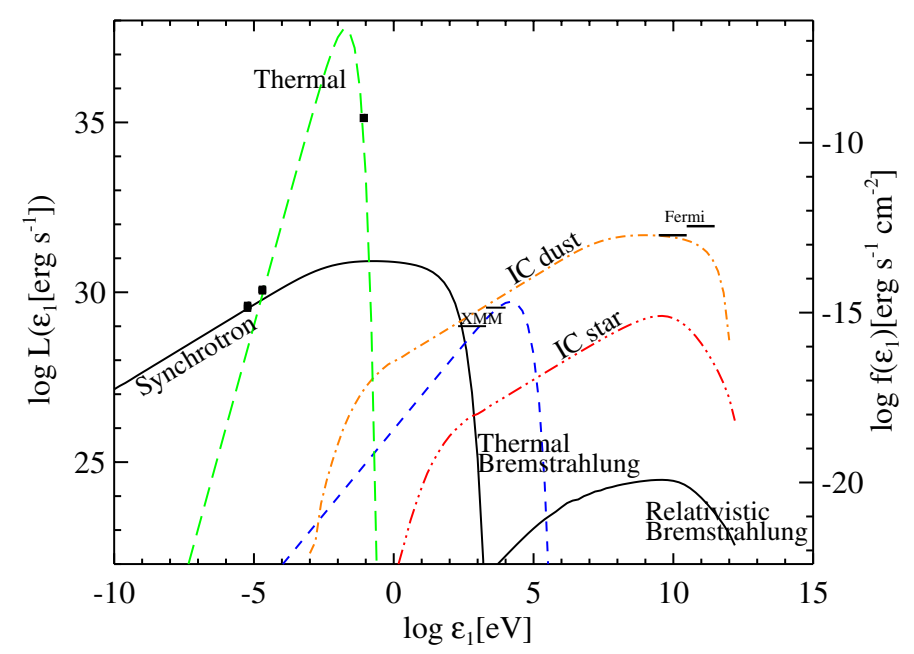

Fig. 4. Luminosity distribution for the bow shock formed by $\mathrm{BD}+433654$. We include the MSK (at about $0.1 \mathrm{eV}$ ) and VLA observations from Benaglia et al. (2010).

longer observations. Up to now, BD+34 3654 was observed with XMM during $47 \mathrm{ks}$, which is not enough to detect the bow shock X-ray emission, according to our simulations. Terada et al. (2012) failed in detecting this bow shock with Suzaku. From their observation, they obtained an X-ray luminosity lower limit at $1.1 \times 10^{32} \mathrm{erg} \mathrm{s}^{-1}$, which is much higher than our predictions.

\subsection{Betelgeuse}

Mackey et al. (2012) developed stellar evolution models and incorporated the evolving stellar wind into hydrodynamic simulations to simulate the transition of Betelgeuse from the blue supergiant (BSG) phase to the red supergiant (RSG) phase at the late stages of its life. At these phases, massive stars may undergo rapid transitions from red to blue supergiants and vice versa in the Hertzprung-Russell diagram, and the stellar wind velocities also change rapidly. Mohamed et al. (2012) found that the bow shock formed by Betelgeuse is still young $(<30 \mathrm{kyr})$. The total kinetic power is rather low, but the star is relatively close and a possible low X-ray luminosity can still be detectable.

During the transient phase from BSG to RSG the defunct wind of the BSG still reaches a few hundred kilometers per second. At the RSG phase the wind velocity decreases to $\sim 20 \mathrm{~km} \mathrm{~s}^{-1}$, and hence the acceleration of particles is inefficient and the nonthermal processes are unable to produce high-energy photons in sufficiently large numbers to be detected with the current X-ray telescopes. Here we used the parameters derived by Mackey et al. (2012) in their simulations to test the possibility of a nonthermal high-energy emission in the transition phase from BSG to RSG, where the kinetic energy of the wind is still high. The result is shown in Fig. 5. The IC of the dust photons can lead to the emission of detectable X-ray photons. We also include the Chandra detection mean limits reported by Posson-Brown et al. (2006) in the $0.1-6 \mathrm{keV}$ range for this star, although they are less sensitive than the XMM-Newton limit reported by Hasinger et al. (2001). Our simulation shows that the most energetic photons could be detected. However, we recall that the current observations of Betelgeuse do not correspond to the transition phase from BSG to RSG, but to the present RSG phase. Hence no detection is expected in the current phase.

Although Betelgeuse is now an RSG star, we used it as a basis to simulate the supergiant stars case and to test the possibility

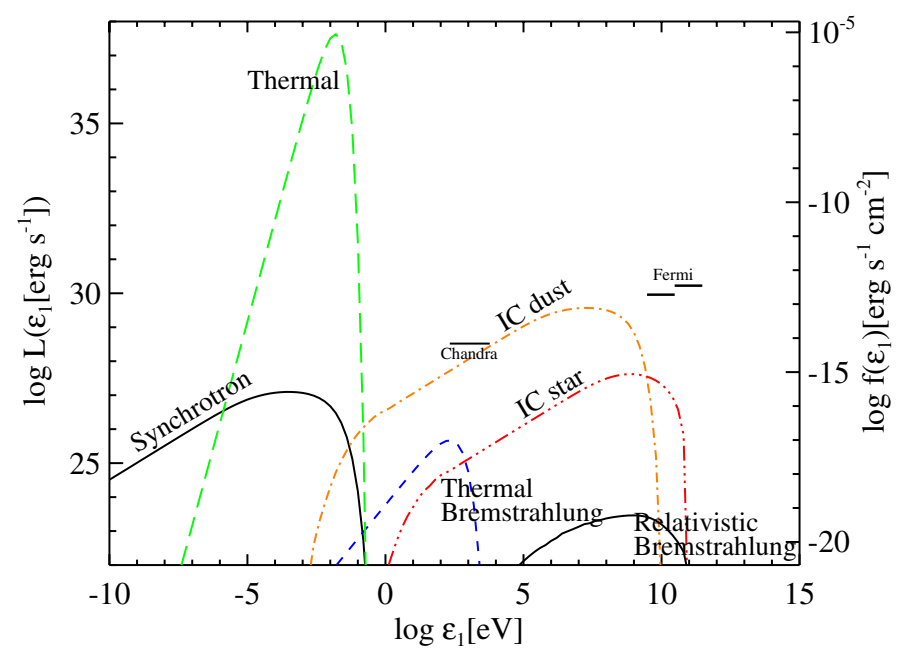

Fig. 5. Luminosity distribution for the bow shock formed by Betelgeuse during the transition phase form blue to red supergiant before the current red supergiant phase.

of nonthermal emission from runaway stars with its characteristics during the transition phase from BSG to RSG. The X-ray luminosity is somehow faint, but it can make up a new source of high-energy photons in the neighborhood of the Earth, although bow shocks formed during the BSG phase could be detected in $\mathrm{X}$-rays at greater distances.

\section{Discussion and conclusions}

We have studied the nonthermal processes that can explain the emission detected from the bow shock formed by the star AE Aur and tested the possibility of other bow shocks to emit highenergy nonthermal photons in sufficiently large numbers to be detected. However, the detection of X-ray emission from bow shocks around runaway stars is not expected to be found around most of them since many scenarios make the formation and detection of the bow shock unlikely. First, the runaway star must move supersonically through the ISM, while low-density mediums do not favor the formation of bow shocks. Second, many runaway stars can form close bow shocks, which can be detectable in the IR, but the resolution of the X-rays telescopes currently available fail in resolving bow shocks with a short standoff radius from their star. Furthermore, the angle formed by the line of sight and the star-bow shock line is of the utmost importance since the bow shock can have been formed far enough from the star but the angle can cause it to be hidden by the runaway star or make it unresolvable from the star. On the other hand, X-ray bow shocks reach luminosities orders of magnitude fainter than the runaway stars that generate them, and hence very long exposure times are needed to detect them.

High mass-loss rates and wind velocities favor the formation of strong shocks that can emit high-energy nonthermal photons. However, they also cause the bow shocks to be formed farther away from the star. The available energy in the acceleration region depends on the relation between the volume of the acceleration region and the volume of a sphere of radius $R_{0}$. Since the acceleration region is a nearly flat region, the available kinetic energy in the acceleration region depends on the distance between the star and the acceleration region, which is equivalent to the standoff radius defined by Eq. (1). The volume of a sphere grows faster with the radius than the volume of the acceleration region, and thus the energy available to accelerate particles 
decreases quickly with the standoff radius. Hence, the energy density is proportional to $R_{0}^{-3}$, and from Eq. (1) the standoff radius is proportional to the inverse of the velocity of the star. These dependencies imply that if the velocity of the star were lower by $20 \%$, the energy density available to accelerate particles would be reduced by $50 \%$. High stellar velocities favor the formation of the bow shock at distances closer to the star, as do high ambient medium densities. Fast winds and high mass-loss rates release great amounts of energy, which allow shocks to be formed at greater distances from the star. However, if these two quantities are very high but are not balanced by a dense medium or by a high velocity of the star, the star can cross the ISM without forming a bow shock $\left(R_{0} \sim \infty\right)$. This emphasizes that the conditions under which a bow shock can be formed by a runaway star, or under which high-energy photons are emitted, are very constrained. It also suggests that only a small fraction of the bow shocks are expected to be detected in the X-ray band. In our three simulations the integrated $\mathrm{X}$-ray flux in the $0.3-10 \mathrm{keV}$ band is lower than $10^{30} \mathrm{erg} \mathrm{s}^{-1}$ (see Figs. 3-5). These low luminosities constrain the search for bright bow shocks to stars closer than $1 \mathrm{kpc}$ because of the limited sensitivity of the X-ray telescopes and the exposure times required. Stars that are located farther away, such as BD+43 3654, demand very long exposure times, but as the emitted X-ray flux from the bow shock is rather low, the background contamination may prevent its detection.

Runaway stars can produce bow shocks when passing through sufficiently dense clouds. These bow shocks can serve as particle acceleration sites where charged particles can cool by nonthermal processes and emit high-energy photons. However, the production of these photons in sufficiently large numbers to be detected is not possible in all bow shocks. Runaway Oand B-type stars moving through dense molecular clouds, with the highest mass-loss rates of all the stars and the highest wind velocities, are considered the most suitable candidates to have formed a high-energy emitting bow shock. However, we showed that runaway supergiant stars in their transition phase from blue supergiant to red supergiant also undergo great losses of mass and have moderate terminal velocities, which makes them a new possible source of X-ray photons.

From all the bow shocks detected around runaway stars, only those formed by the stars AE Aur and BD+43 3654 have been directly observed in X-rays (although the bow shock formed by BD+43 3654 has not been detected). For AE Au we succeeded in reproducing the nonthermal emission from the shock. For the bow shock formed by BD+433654 we also obtained the luminosity distribution and showed that it emits high-energy photons, but the fluxes are too low to be detected. More observations are needed to constrain the stellar parameters that favor the shock formation and can lead to the production of enough high-energy photons to be detectable in the X-ray domain, but our model sets a theoretical scenario to constrain the most reliable candidates to produce high-energy emission and to test whether they may be detectable.
We showed that bow shocks formed by different types of runaway stars can account for a significant fraction of the highenergy photons produced in our Galaxy. More energetic events in our galaxy, such as supernova remnants, produce a larger amount of high-energy photons, and these sources can be detected at larger distances. However, the IC losses undergone by the electrons accelerated in the bow shock regions formed by runaway stars are revealed to be a considerable source of X-rays and gamma rays in our neighborhood.

Acknowledgements. This work was supported by the Spanish Ministerio de Economía y Competitividad under grant AYA2011-29754-C03-03.

\section{References}

Adams, D. J. 1980, Cosmic X-ray astronomy (Bristol: Adam Hilger, Ltd.) Acero, F., Lemoine-Goumard, M., Renaud, M., et al. 2015, A\&A, 580, A74 Benaglia, P., Romero, G. E., Martí, J., Peri, C. S., \& Araudo, A. T. 2010, A\&A, 517, L10

Blaauw, A. 1961, Bull. Astron. Inst. Netherlands, 15, 265

Comerón, F., \& Pasquali, A. 2007, A\&A, 467, L23

del Valle, M. V., \& Romero, G. E. 2012, A\&A, 543, A56

del Valle, M. V., \& Romero, G. E. 2014, A\&A, 563, A96

Fullerton, A. W., Massa, D. L., \& Prinja, R. K. 2006, ApJ, 637, 1025

Gvaramadze, V. V., \& Bomans, D. J. 2008, A\&A, 485, L29

Gies, D. R. 1987, ApJS, 64, 545

Gies, D. R., \& Bolton, C. T. 1986, ApJS, 61, 419

Ginzburg, V. L., \& Syrovatskii, S. I. 1964, The Origin of Cosmic Rays (New York: Macmillan)

Hasinger, G., Altieri, B., Arnaud, M., et al. 2001, A\&A, 365, L45

Hoogerwerf, R., de Bruijne, J. H. J., \& de Zeeuw, P. T. 2000, ApJ, 544, L133

Howarth, I. D., Siebert, K. W., Hussain, G. A. J., \& Prinja, R. K. 1997, MNRAS, 284,265

Hubrig, S., Oskinova, L. M., \& Schöller, M. 2011, Astron. Nachr., 332, 147

Kelner, S. R., Aharonian, F. A., \& Bugayov, V. V. 2006, Phys. Rev. D, 74, 034018 Kobulnicky, H. A., Gilbert, I. J., \& Kiminki, D. C. 2010, ApJ, 710, 549

Longair, M. S. 2011, High Energy Astrophysics, ed. M. S. Longair (Cambridge, UK: Cambridge University Press)

López-Santiago, J., Miceli, M., del Valle, M. V., et al. 2012, ApJ, 757, L6

Mackey, J., Mohamed, S., Neilson, H. R., Langer, N., \& Meyer, D. M.-A. 2012, ApJ, 751, L10

Maíz-Apellániz, J., Walborn, N. R., Galué, H. Á., \& Wei, L. H. 2004, ApJS, 151, 103

Markova, N., Puls, J., Repolust, T., \& Markov, H. 2004, A\&A, 413, 693

Mohamed, S., Mackey, J., \& Langer, N. 2012, A\&A, 541, A1

Moffat, A. F. J., Marchenko, S. V., Seggewiss, W., et al. 1998, A\&A, 331, 949

Moffat, A. F. J., Marchenko, S. V., Seggewiss, W., et al. 1999, A\&A, 345, 321

Peri C.S., Benaglia, P., Brookes, D. P., Stevens, I. R., \& Isequilla, N. L. 2012, A\&A, 538, A108

Posson-Brown, J., Kashyap, V. L., Pease, D. O., \& Drake, J. J. 2006, ArXiv e-prints [arXiv:astro-ph/0606387]

Repolust, T., Puls, J., \& Herrero, A. 2004, A\&A, 415, 349

Rybicki, G. B., \& Lightman, A. P. 1979 (New York, Wiley-Interscience), 393

Terada, Y., Tashiro, M. S., Bamba, A., et al. 2012, PASJ, 64, 138

Tetzlaff, N., Neuhaeuser, R., \& Hohle, M. M. 2010, VizieR Online Data Catalog: J/MNRAS/410/190

van Buren, D., \& McCray, R. 1988, ApJ, 329, L93

Vink, J. S., de Koter, A., \& Lamers, H. J. G. L. M. 2001, A\&A, 369, 574

Wilkin, F. P. 1996, ApJ, 459, L31 